\section{Prevalence of chronic disabling fatigue in children}

\section{and adolescents}

ANNE FARMER, TOM FOWLER, JANE SCOURFIELD and ANITA THAPAR

\author{
Background The epidemiology of \\ chronic fatiguing illnesses in young people \\ is poorly understood.
}

\begin{abstract}
Aims To estimate the lifetime prevalence of different definitions of chronic fatigue in 8- to 17-year-olds.
\end{abstract}

\begin{abstract}
Method Participants came from two population-based twin series. Parents completed self-report questionnaires that enquired whether either child had ever experienced more than a few days of disabling fatigue. Telephone interviews were undertaken for individuals who had experienced such an episode.
\end{abstract}

Results Questionnaires were returned by 1468 families (65\% response rate) and telephone interviews were undertaken regarding 99 of the 129 subjects (77\%) who had experienced fatigue. The lifetime prevalence estimates ranged from 2.34\% (95\% Cl I.75-2.94) for disabling fatigue lasting 3 months to $1.29 \%(95 \% \mathrm{Cl}$ 0.87-1.7I) for a disorder resembling adult operationally defined chronic fatigue syndrome.

Conclusions From the age of II years, young people have similar rates and types of chronic fatiguing illnesses to adults.

Declaration of interest Funded by a grant from the PPP Charitable Trust (A.F. and A.T.).
Chronic fatiguing disorders occurring in adolescence can cause considerable morbidity and severe impairment of educational attainment and social development (Dowsett \& Colby, 1997). In a workshop report, Marshall (1999) noted the lack of epidemiological studies in this age group and pointed to the need for populationbased samples that avoid referral and treatment-seeking biases. Other recent reviews highlight the lack of research in this age group compared with adults (Garralda \& Rangel, 2001). In particular, there is little agreement about the prevalence of disabling fatigue in young people or whether the narrow definitions of chronic fatigue syndrome (CFS) devised to standardise diagnosis in adult populations (Fukuda et al, 1994) are relevant to these younger age groups (Marshall, 1999). In the current study we have used two general populationbased twin series to derive lifetime prevalence estimates of different definitions of chronic fatigue in 8 - to 17 -year-olds.

\section{METHOD}

\section{Participants}

The participants were identified from two population-based twin registers based in South Wales and Greater Manchester. These registers have been amalgamated recently to form the 'Cardiff Study of All Wales and Northeast England Twins' twin register (CASTANET). Both registers were set up from birth registers and were originally created from community child health databases. Initially, twin pairs were excluded if one or both twins had severe learning disability, severe physical handicap or if one of the twins had died. Previous studies have shown that the registers are representative of the local population (Scourfield et al, 1999; Thapar et al, 2000).

The combined registers consisted of 3051 pairs of twins aged 8-17 years. The general practitioners of all the twins were first contacted to check for any contraindications to contact by the study team, which led to 34 families not being approached. A further 748 families could not be located at the address listed on the register and further efforts to verify the current address held by a British Telecom database or the electoral register proved unsuccessful. Consequently, the final sample consisted of 2269 twin pairs, of whom $29.8 \%$ came from South Wales and $70.2 \%$ from Manchester.

\section{Measures}

The parents were sent questionnaires relating to each child separately, to complete and return by post. Three reminder letters and a further set of questionnaires were sent to non-responders 4 months after the first mailing.

The questionnaires enquired whether the child had ever experienced a period of tiredness lasting at least several days that had caused interference with activities such as being able to go to school or see friends or family. If they answered 'yes' to this question, they were asked to indicate the duration of the disabling fatigue on a fivepoint scale, ranging from 'a few days' to 'more than 6 months'. Parents were also asked to give written consent to further contact by telephone.

Those who agreed to further contact and where one or both children had experienced more than a few days of fatigue were interviewed by telephone. For those over the age of 11 years, both the main carer and the young person were interviewed. For younger participants, only the parent/main carer was interviewed. In this study we report on the results of the parents' interviews.

The interview consisted of two parts. The first part obtained further details about the period(s) of fatigue, including the type and degree of impairment in functioning as well as information to determine whether the young person had experienced a similar symptom profile, duration and impairment to the Centers for Disease Control operational criteria for CFS in adults (Fukuda et al, 1994). The second part of the interview consisted of the depression section of the Child and Adolescent Psychiatric Assessment (Angold \& Costello, 1995).

Information was sought about the duration of the fatigue and whether it had 
been continuous or episodic. The nature and degree of impairment associated with the fatigue was assessed by enquiring about whether the twin needed to rest for at least 1 hour daily, whether there was interference with school attendance, and how the tiredness interfered with the subject's usual activities and family and peer relationships. With regard to school attendance, the parent was asked whether the twin needed to take time off from school and whether the amount of time off from school meant that the twin had to repeat a school year, catch up in the school holidays, have a home tutor, have work sent home by teachers or have a reduced timetable. Parents were also asked about the number of visits to general practitioners and/or hospital specialists made regarding the fatigue. The interviewer also recorded verbatim the diagnoses that the parent reported had been made by the general practitioner and/or hospital specialist. The physician diagnoses were reviewed by an independent clinician who determined those cases where the diagnosis could entirely explain the chronic fatigue in the twin. These cases were then excluded from further analysis. In addition, the parent was also asked what he or she thought was the cause of the fatigue. These responses were also recorded verbatim.

To assess whether the young person's fatigue resembled that found in adults and fulfilled the adult Centers for Disease Control criteria for CFS (Fukuda et al, 1994), enquiry was made about symptoms that sometimes occur with fatigue. These were lack of energy, need for rest, unrefreshing sleep, muscle pain, eye strain, poor memory and concentration, 'slips' of the tongue, difficulty in initiating activities, fatiguability, headaches, multiple joint pains, tender lymph nodes, feeling weak, sore throats, difficulty thinking or postexertion malaise associated with the period of fatigue. These items were rated as 'less than usual', 'same as usual', 'more than usual' or 'a lot more than usual' for the episode of chronic fatigue.

The second part of the telephone interview consisted of the depression section of the Child and Adolescent Psychiatric Assessment (Angold \& Costello, 1995), a semi-structured investigator-based research diagnostic interview. The responses to this were used to apply the DSM-IV (American Psychiatric Association, 1994) operational criteria for major depression for the episode of fatigue.

\section{Analyses}

The population-based twin pairs can be considered as independently sampled, but each pair contributes two sets of observations that cannot be classed as in dependent (Bland \& Kerry, 1997). Consequently, the survey analysis procedures of Stata Release 6 for PC were used to adjust the variances of the analyses, which were used in versions of independent $t$-tests and $\chi^{2}$ tests.

Because not all families where there were positive replies to the screening fatigue questions wished to be interviewed, a mid-range estimate of prevalence was calculated that assumed that the same proportion of children fulfilled the different definitions of chronic fatigue in those from families who did not agree to participate as among those who did.

\section{RESULTS}

\section{Response rates}

Parents from 1468 families returned questionnaires, which is a response rate of $65 \%$. There were no significant sociodemographic differences between the responding and non-responding families (Rice et al, 2002).

One hundred and twenty-nine young people $(4.4 \%)$ were identified by the screening questionnaires as having experienced more than a few days of disabling fatigue, and telephone interviews were undertaken for 99 (77\%). Of those not interviewed, 15 had indicated that they did not wish to participate and a further 15 failed to respond to written requests for telephone contact. Following the interview, three young people who had diagnoses of cerebral palsy, nephrotic syndrome and thalassaemia were also excluded following independent paediatric advice that these disorders could entirely explain the fatigue. Thus, the following results are based on 96 young people (see Fig. 1).

\section{Age at interview, gender and location}

For 12 families both twins were reported as having experienced disabling fatigue, whereas for 72 families only one twin was reported as having experienced disabling fatigue. Thirty-one $(32 \%)$ of the twins came from South Wales whereas 65 $(68 \%)$ came from Greater Manchester. Two-thirds (64) of the fatigued twins were female and one-third (32) were male. The mean age at interview was 14 years 8 months (range: 9 years 5 months to 18 years 5 months).

\section{Characteristics of fatigue}

The mean age of onset of fatigue in the 32 boys was 11 years 1 month (s.e. $=7.93$ months) and for the 64 girls it was 11 years 3 months (s.e. $=6.23$ months). There were no significant gender differences for age at onset.

The mean duration of fatigue in the 32 boys was 9.18 months (s.e. $=5.15)$ and for the 64 girls it was 13.84 months (s.e.=3.51). There were no significant gender differences for duration of fatigue.

\section{Other symptoms associated with prolonged fatigue}

Table 1 shows that the most common associated symptoms were lacking energy, needing to rest, multiple joint pain and unrefreshing sleep. The Centers for Disease Control criteria for CFS in adults (Fukuda et al, 1994) require the presence of at least four out of eight minor symptoms concomitant with the period of fatigue (these eight

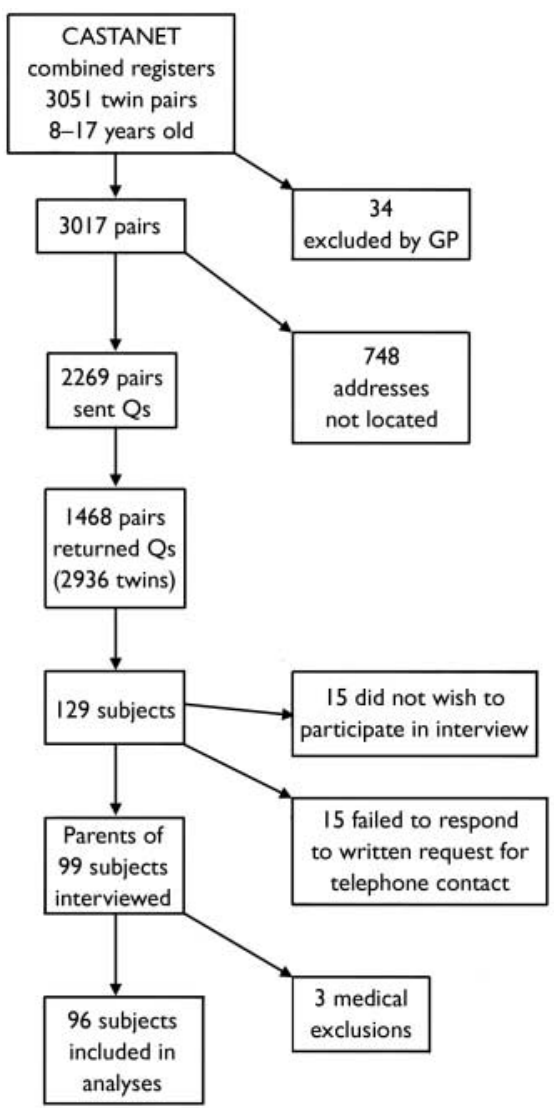

Fig. I Flow chart of subject inclusions and exclusions in the study (Qs, questionnaires; GP. general practitioner) 
symptoms are indicated by asterisks in Table 1). A total of $86 \%$ of boys and $77 \%$ of girls had at least four concomitant symptoms.

\section{Educational and social impairment}

A total of 14 boys (44\%) and 34 girls $(53 \%)$ had to have time off from school because of the fatigue and for 9 boys $(28 \%)$ and 24 girls $(38 \%)$ this interference with their education was more severe, requiring extra work in the holidays, work sent home by teachers, a home tutor or a reduced timetable. Boys missed a mean of 9.50 days (s.e. $=2.22$ ) and girls missed a mean of 14.62 days (s.e. $=1.97$ ) per term. Parents of all 96 subjects reported that chronic fatigue had caused impaired relationships with family and friends and reduced participation in leisure activities.

\section{Visits to doctors}

General practitioners were consulted about the fatigue for 22 boys $(67 \%)$ and 44 girls $(67 \%)$. For $12(36 \%)$ boys and $24(36 \%)$ girls a hospital specialist opinion was also sought. For the boys, the mean number of visits to the general practitioner was 2 $($ s.e. $=0.26)$ and for girls it was 4.35 (s.e. $=0.75)$. Girls had significantly more visits to their general practitioners compared with boys $(t=-2.96$, d.f. $=64$, $P=0.005)$. In 22 cases general practitioners considered that the tiredness was due to some type of infection, including glandular fever (five cases), but in 21 cases they failed to give an explanation for the fatigue according to the interviewed parent. Psychological causes, age, hormones or puberty were given as causes of the fatigue in nine subjects whereas single causes were given for the remainder. Hospital specialists were more likely to have given a diagnosis for the cause of the fatigue and there was uncertainty only in five cases. Psychological or psychiatric causes were given in five cases and specific infections in four. The general practitioners and specialists were reported to have carried out 42 blood tests, of which eight had positive findings (four indicated an infection and four indicated anaemia). Only in one case was a diagnosis of CFS made by a hospital specialist (a full table of the diagnoses given and tests undertaken by general practitioners and hospital specialists can be obtained from the authors on request). The commonest parental attribution for fatigue in their twin was a psychological cause $(28 \%$ of fatigued twins)
Table I Frequencies of symptoms associated with prolonged fatigue in boys and girls

\begin{tabular}{|c|c|c|}
\hline Symptom & $\begin{array}{l}\text { Frequency } \\
\text { (\%) in boys }\end{array}$ & $\begin{array}{l}\text { Frequency } \\
\text { (\%) in girls }\end{array}$ \\
\hline Lack energy & 94 & 86 \\
\hline Rest more & 85 & 88 \\
\hline Multiple joint pain* & 85 & 78 \\
\hline Unrefreshing sleep* & 85 & 73 \\
\hline $\begin{array}{l}\text { Post-exertional } \\
\text { malaise* }\end{array}$ & 76 & 69 \\
\hline $\begin{array}{l}\text { Difficulty starting } \\
\text { things }\end{array}$ & 76 & 69 \\
\hline Poor concentration* & 76 & 64 \\
\hline Headaches* & 64 & 65 \\
\hline Feel weak & 52 & 61 \\
\hline Fatiguability & 45 & 42 \\
\hline Muscle pain* & 39 & 39 \\
\hline Tender lymph nodes* & 39 & 52 \\
\hline Difficulty thinking & 33 & 45 \\
\hline Sore throats* & 27 & 45 \\
\hline Slips of the tongue & 24 & 22 \\
\hline Poor memory & 18 & 23 \\
\hline Eyestrain & 18 & 34 \\
\hline
\end{tabular}

Associated symptoms required for the Centers for Disease Control criteria for chronic fatigue syndrome in adults are indicated by asterisks (Fukuda et al, 1994). There are no significant gender differences for any of the symptoms associated with chronic fatigue.

whereas a further $20 \%$ of parents thought that their twin's fatigue was due to an infection. Chronic fatigue syndrome or myalgic encephalomyelitis was not attributed as a cause by any parent (a full table of all the causes cited by parents for fatigue in the twins can be obtained from the authors on request).

\section{Depression and chronic fatigue}

Twelve boys (38\%) and 25 girls (39\%) fulfilled the DSM-IV operational definitions for mild, moderate or severe depression during the episode of fatigue. There were no gender differences for concomitant depression. Table 2 shows that the percentage of subjects with co-occurring depression rises as the duration and severity of the fatigue increases.

\section{Numbers of subjects fulfilling different definitions of chronic fatigue and CFS}

Table 2 shows that of the 32 boys and 64 girls with more than a few days of chronic fatigue, for 19 boys and 50 girls this lasted for at least 1 month. For 17 boys (53\%) and 44 girls $(67 \%)$ the chronic fatigue had lasted at least 3 months, of whom 14 boys $(44 \%)$ and 36 girls $(55 \%)$ had also had at least four concomitant symptoms. Nine boys $(28 \%)$ and 26 girls $(41 \%)$ experienced fatigue lasting 6 months with at least four concomitant symptoms. There were no gender differences for these different severities of fatigue. Table 2 also shows that the mean ages of onset for these different durations of fatigue ranged from 11.13 years to 12.43 years, and that between onethird and a half of the subjects also fulfilled DSM-IV criteria for depression during the period of chronic fatigue.

\section{Prevalence of different definitions of chronic fatigue}

The estimated prevalence of fatiguing illness lasting 3 months or more was $2.34 \%$ (95\% CI 1.75-2.94) whereas the prevalence of fatigue lasting 3 months accompanied by at least four minor symptoms from the Centers for Disease Control criteria for CFS was $1.90 \% \quad(95 \%$ CI $1.40-2.40)$. Lastly, the prevalence of 6 months or more of fatigue with at least four minor symptoms was $1.29 \%$ (95\% CI 0.87-1.71).

\section{DISCUSSION}

The present study has been undertaken in twin samples that have been shown to be representative of the general populations of South Wales and Greater Manchester. Because there is no evidence that twins are either more or less susceptible to chronic fatigue or CFS than singletons, it is reasonable to use the CASTANET twin sample to derive population prevalence estimates (Thapar et al, 2000; Rice et al, 2002).

\section{Frequency, duration and age of onset of chronic disabling fatigue in young people}

The results show that disabling fatigue in young people is not rare and that $4.4 \%$ of the parents who responded to a questionnaire survey reported the symptom lasting for more than a few days in one or both of their children. However, this is less than half the rate in adults where estimates for the rates of 'persistent fatigue' complaints to primary care physicians range from $11 \%$ to $27 \%$ (Wessely et al, 1997 ; Skapinakis et al, 2000; Sharpe \& Wilks, 2002). The 
Table 2 Number of fatigued boys and girls, mean age at onset and \% with comorbid depression for five categories: any duration, at least I month, 3 months or more, 3 months and at least four minor symptoms and 6 months or more and at least four minor symptoms

\begin{tabular}{|c|c|c|c|c|c|c|}
\hline $\begin{array}{l}\text { Duration of fatigue } \\
\text { ( } \pm 4 \text { minor symptoms) }\end{array}$ & No. of boys & No. of girls & $\begin{array}{c}\text { Age at onset in months } \\
\text { in boys (s.e.) }\end{array}$ & $\begin{array}{l}\text { Age at onset in months } \\
\text { in girls (s.e.) }\end{array}$ & $\begin{array}{l}\text { \% Boys with } \\
\text { depression' }\end{array}$ & $\begin{array}{l}\text { \% Girls with } \\
\text { depression' }\end{array}$ \\
\hline More than a few days & 32 & 64 & $133.50(7.93)$ & $|35.4|(6.23)$ & $38 \%$ & $39 \%$ \\
\hline At least I month & 19 & 50 & $143.84(10.71)$ & I36.42 (6.99) & $42 \%$ & $46 \%$ \\
\hline 3 months & 17 & 44 & $144.82(\mid 1.70)$ & I36.75 (7.72) & $41 \%$ & $48 \%$ \\
\hline 3 months $( \pm 4)$ & 14 & 36 & $|49.2|(8.75)$ & $134.78(9.09)$ & $36 \%$ & $53 \%$ \\
\hline 6 months $( \pm 4)$ & 9 & 26 & |48.22 (10.99) & 140.38 (II.22) & $56 \%$ & $50 \%$ \\
\hline
\end{tabular}

I. Percentage of subjects fulfilling the DSM-IV criteria for major depression for the period of fatigue.

study showed that the mean duration of chronic fatigue (9.57 months for boys and 13.49 months for girls) is similar to that reported in studies of CFS in paediatric clinic samples. For example, Carter et al (1995) report a mean duration of 11 months in 20 individuals whose mean age was 13 years, whereas Feder et al (1994) report a mean duration of 7 months in 48 young people whose mean age was 15 years. However, the individuals in the present study were younger than these specialist clinic referrals, with the mean age at onset for both genders around 11 years. This is very similar to that reported in a telephone survey of CFS-like illnesses in children in Chicago where the mean age at onset was 11.6 years (Jordan et al, 2000). Thus, community surveys such as the present study appear to identify fatiguing illness of the same duration as those reported from specialist clinical services, although the children are on average at least 2 years younger.

Our study confirms the findings from other studies that chronic fatigue is rare in children under 10 years old (Marshall, 1999). In addition, the gender ratio of approximately twice as many girls $(67 \%)$ as boys $(33 \%)$ reported with the symptom is also similar to other studies both of children and of adults (Carter et al, 1995; Wessely et al, 1997).

\section{Relationship of chronic fatigue in the twins to operationally defined CFS}

Following the telephone interview we have found that chronic fatigue of the same duration, impairment and number of associated symptoms as the adult Centers for Disease Control operational definition of CFS (Fukuda et al, 1994) has an estimated lifetime prevalence of $1.29 \%$, whereas for a broader definition, including those with 3 months of symptom duration, a lifetime prevalence of $1.90 \%$ was found. The prevalence estimates for these 'broad' ( 3 months' duration) and 'narrow' (6 months' duration) definitions of chronic fatigue are not much different, which supports the view that the diagnosis of CFS in this younger age group should be made after 3 months of symptoms with impairment rather than after 6 months as the adult definition requires (Royal Colleges of Physicians, Psychiatrists and General Practitioners, 1996).

Despite not being formally diagnosed with CFS, the gender ratio (twice as many girls as boys), number and type of associated symptoms, frequency of co-occurring depression (just under $40 \%$ ) and duration and impairment of chronic fatigue are little different in our population-based sample compared with those reported for clinical samples of young people diagnosed with CFS (Feder et al, 1994; Carter et al, 1995; Garralda \& Rangel, 2001). It is also noteworthy that the percentage of subjects with co-occurring depressive disorder also increased with increasing duration of their chronic disabling fatigue.

\section{Comparisons with another recently published epidemiological study of chronic fatigue in young people}

Our prevalence estimates based on parental accounts are somewhat higher than the rates reported by Chalder and colleagues who undertook telephone interviews with 4240 children aged 11-15 years as part of a large epidemiological study of mental health in this age group (Chalder et al, 2003). They report a chronic fatigue prevalence of $0.57 \% \quad(95 \%$ CI $0.34-0.80)$ whereas we have a 'narrow' definition prevalence of $1.29 \%$ (95\% CI $0.87-1.71$ ).
However, from the published account of their study it would appear that they have determined point prevalence whereas in our study we have presented lifetime prevalence estimates.

\section{Limitations of the study}

Our study has a number of limitations. First, the results are based on an interview with a parent and not the child. Second, the interviewer was not a clinician so medical exclusions were based on obtaining a verbatim account of what the parent reported a doctor had said was the cause of fatigue in the twin and the advice of an independent clinician that the medical condition listed could entirely explain the chronic fatigue. Other conditions that could have partly explained the fatigue (e.g. viral infections) but not its severity or duration were retained in the analyses. Finally, because of the absence of a clinical examination of the twins, we have only reported on the resemblance of our subjects to CFS as defined by the Centers for Disease Control in terms of morbidity rather than the subjects fulfilling these criteria. Despite these caveats, our study has shown that the symptom pattern and severity, duration and associated impairment associated with chronic fatigue in young people are similar to those found in studies of adults with CFS (Farmer et al, 1996; Wessely et al, 1997) and that prevalence estimates also fall within the range of $0.07-2.6 \%$ reported in studies of chronic fatigue in adults (Bates et al, 1993; Buchwald et al, 1995; Wessely et al, 1997).

\section{REFERENCES}

American Psychiatric Association (1994) Diagnostic and Statistical Manual of Mental Disorders (4th edn) (DSM-IV).Washington, DC: APA. 
Angold, A. \& Costello, E. J. (1995) A test-retest reliability study of child-reported psychiatric symptoms and diagnoses using the Child and Adolescent Psychiatric Assessment (CAPA-C). Psychological Medicine, 25, $755-762$

Bates, D. Schmiddt, W., Lee, J., et al (1993) Prevalence of fatigue and chronic fatigue syndrome in primary and tertiary care. Archives of Internal Medicine, I53, 2759-2765.

Bland, J. M. \& Kerry, S. M. (1997) Statistics notes. Trial randomised into clusters. BMJ, 315, 600.

Buchwald, D., Umali, P., Umali, J., et al (1995)

Chronic fatigue and chronic fatigue syndrome:

prevalence in a Pacific Northwest health care system.

Annals of Internal Medicine, 123, 81-88.

Carter, B. D., Edwards, J. F., Kronenberger, W. G., et al (1995) Case control studies of chronic fatigue in pediatric patients. Pediatrics, 95, 179-186.

\section{Chalder, T., Goodman, R., Wessely, S., et al (2003)}

Epidemiology of chronic fatigue syndrome and self reported myalgic encephalomyelitis in 5-15 year olds; a cross sectional study. BMJ, 327, 654-655.

Dowsett, E. G. \& Colby, J. (1997) Long term sickness absence due to ME/CFS in UK schools: an epidemiological study with medical and educational implications. Journal of Chronic Fatigue Syndrome, 3, 29-42.

Farmer, A., Chubb, H., Jones, l., et al (1996) Screening for psychiatric morbidity in subjects presenting with chronic fatigue syndrome. British Journal of Psychiatry, 168, 354-358.

Feder, H. M., Dworkin, P. H. \& Orkin, C. (1994) Outcome of 48 pediatric patients with chronic fatigue: a clinical experience. Archives of Family Medicine, 3. 1049-1055.

Fukuda, K., Straus, S. E., Hickie, l., et al (1994) The chronic fatigue syndrome: a comprehensive approach to its definition and study. Annals of Internal Medicine, I2I, 953-959.

Garralda, M. E. \& Rangel, L. (200I) Childhood chronic fatigue syndrome (letter). American Journal of Psychiatry, 158, I|61.

Jordan, K. M., Ayers, P. M., Jahn, S. C., et al (2000) Prevalence of fatigue and chronic fatigue syndrome like illness in children and adolescents. Journal of Chronic Fatigue Syndrome, 6, 3-21.

Marshall, G. S. (1999) Report of a workshop on the epidemiology, natural history and pathogenesis of chronic fatigue syndrome in adolescents. Journal of Pediatrics, 134, 395-405.

Rice, F., Harold, G. T. \& Thapar, A. (2002) Assessing the effects of age, sex and shared environment on the genetic aetiology of depression in childhood and

\section{CLINICAL IMPLICATIONS}

- From the age of II years, young people have the same prevalence, symptom profile, gender distribution, co-occurring depression and severity of fatiguing disorders as do adults.

- Chronic fatigue causes considerable impairment in young people, to both social development and time missed from school.

- Operational criteria for chronic fatigue syndrome (CFS) should require only 3 months' symptom duration in young people and not 6 months as in adults.

\section{LIMITATIONS}

- Interviews were carried out by telephone, not face to face.

- The study presents parental accounts of fatigue rather than young people's own accounts.

- Interview questions were not validated in CFS clinic attenders or control subjects.

ANNE FARMER, FRCPsych, MRC Social, Genetic, Developmental Psychiatric Research Centre, Institute of Psychiatry, London; TOM FOWLER, PhD, JANE SCOURFIELD, MRCPsych, ANITA THAPAR, Department of Psychological Medicine, University of Wales College of Medicine, Cardiff

Correspondence: Professor Anne Farmer, MRC Social, Genetic, Developmental Psychiatric Research Centre, Institute of Psychiatry, De Crespigny Park, Camberwell, London SE5 8AF, UK. Tel: +44 (0) 207 848 0940; fax: +44 (0) 207848 0856; e-mail: spjuaef@iop.kcl,ac.uk

(First received 14 August 2003, final revision 23 January 2004, accepted I February 2004)

adolescence. Journal of Child Psychology and Psychiatry, $\mathbf{4 3}$ |039-105|

\section{Royal Colleges of Physicians, Psychiatrists and} General Practitioners (1996) Chronic Fatigue Syndrome. Report of a Joint Working Group (Council Report CR54). London: Royal College of Physicians Publications Unit.

Scourfield, J., Martin, N., Lewis, G., et al (1999) Heritability of social cognitive skills in children and adolescents. British Journal of Psychiatry, 175, 559-564.

Sharpe, M. \& Wilks, D. (2002) Fatigue. BMJ, 325, 480-483.
Skapinakis, P., Lewis, G. \& Meltzer, H. (2000)

Clarifying the relationship between unexplained chronic fatigue and psychiatric morbidity: results from a community survey in Great Britain. American Journal of Psychiatry, I57, 1492-1498.

Thapar, A., Harrington, R., Ross, K., et al (2000) Does the definition of ADHD affect heritability? Journal of the American Academy of Child and Adolescent Psychiatry, 39, 1528-1536.

Wessely, S., Chalder, T., Hirsch, S., et al (1997) The prevalence and morbidity of chronic fatigue syndrome: prospective primary care study. American Journal of Public Health, 87, 1449-1455. 